\title{
El hombre, como «memoria Dei», en el pensamiento de Paul Tillich
}

\author{
El hombre, «memoria Dei», por razón de su mismo ser
}

Los que se han ocupado de la memoria Dei como presencia de Dios en el alma, han limitado generalmente su contenido al aspecto de la teoría del conocimiento. Pero la verdad es que, tratándose del hombre, parece que habría que decir que, más que existir en él una memoria Dei, es él mismo una memoria Dei. Es decir, el problema de la memoria Dei en el hombre tiene mucho que ver con el problema de la trascendencia e inmanencia de Dios en la creación.

Sabido es que al final de la Edad Media aparecen algunos filósofos en el Renacimiento, a partir de Nicolás de Cusa, que se plantean un problema muy serio para el creyente intelectual, al proponerse éste establecer la relación entre la trascendencia de Dios y su inmanencia en sus criaturas. Esta preocupación era muy legítima, como lo es toda búsqueda de la verdad, pero de hecho resultó peligrosa y de consecuencias funestas para el futuro; nos referimos a un racionalismo que tiene lugar en la Edad Moderna de la filosofía que, en su peor aspecto, se tradujo en los panteísmos de Böhme, Spinozza y, finalmente, del panteísmo alemán tan conocido.

Esto fue, sin duda, una tragedia respecto del problema de Dios en su relación ontológica con las criaturas; pero se ha hablado también de otra tragedia a causa del triunfo de la corriente aristotélico-tomista. El profesor Garrido, siguiendo a Dourlay, nos recuerda lo que fue la clave de esta tragedia con estas palabras: "O se admite la teoría de la participación agustiniano-franciscana, o nos echamos en brazos de Aristóteles. Esta participación que aparece como fondo del problema del conocimiento es en realidad un problema ontológico. Tillich entiende que la tradición aristotélico-tomista ha negado esa participación defendida por Agustín y Buenaventura, perdiendo de esta manera el sentido de la presencia perceptible de Dios a la mente y perdiendo a la vez el sentido sacramental de la naturaleza. Por el contrario, la nueva ontología de matiz aristotélico ofrece una mayor autonomía a la mente y favorece el estudio de las ciencias naturales; creando a su vez una distancia abismal entre Dios y el hombre. Aparece un Dios lejano que se relaciona con el hombre «desde 
afuera»; lo cual prodúce toda una heteronomía religiosa tanto a nivel doctrinal como a nivel ético" ' '. Esta tragedia podría resumirse en la contraposición de Dios como Ipsum Esse del cual participamos - concepción agustinianofranciscana - y el Dios «persona» ante el que nos encontramos como con un "otro», $y$, por lo mismo, como negándole autenticidad. $Y$ así, si la primera postura pudo llevar en su extremismo al panteísmo, la segunda dio ocasión a un punto débil, la autonomía, sin su relación auténtica de participación del /psum Esse que, en el Medioevo se resuelve en un pietismo eclesiástico de marcado carácter heterodoxo, con "conclusiones lógicamente correctas», pero que «tenían que ser avaladas por la autoridad». Sin duda la contraposición hecha por Tillich de estas dos posturas da pie para pensar que si la postura de los filósofos del Renacimiento, de mentalidad agustiniano-franciscana, abrió un portillo a un futuro panteísta, la postura aristotélico-tomista, una vez dejada a un lado por otros filósofos la autoridad, parecería haber abierto otro a racionalismos posteriores. Tillich nos dirá sin más que «la divergencia entre estos dos modos de acercarse a Dios es el gran problema de la filosofía de la religión, y... también la causa principal de la laicización del mundo occidental». Hubo en Tomás un "gap», nos dice Tillich, una especie de vacío entre lo finito y el Infinito entre los que sólo puede haber analogía, un punto débil que sólo saldrá a luz con Duns Scoto. En estas circunstancias, sigue diciendo Tillich, «la Iglesia garantiza la verdad que no puede ser alcanzada mediante un puro acercamiento empírico a Dios ${ }^{2}$. Otra solución fue un secularismo autónomo sin sustancia religiosa ${ }^{3}$.

Los filósofos escolásticos, en su tentativa sin duda auténtica por salvaguardar la infinitud de Dios en su trascendencia y a la vez en su inmanencia, a base sobre todo, de la revelación del hecho creador, parecerían ofrecer a la imaginación una especie de ubicación, de "colocación» de Dios «al lado» o "entre» las cosas creadas. De ahí que el de Cusa, por ejemplo, tienda a acercar lo finito al infinito con su famosa frase de "coincidencia de opuestos», que

1. A. GARRIDO, «Autonomía o heteronomía. La solución de Paul Tillich», en Estudio Agustiniano 12(1977)563.

2. "The divergence between these two approaches to the knowledge of God is the great probleme of the philosphy of religion, and, as I will now show, it is the ultimate cause of the secularization of the western world... The Aristotelian method is placed against the Augustinian, and gradually this method of starting with the external world prevailed. Thomas knew that the conclusions reached in this way, though they are logically correct, do not produce a real conviction about God. Therefore, they must be completed by authority. In other words, the church guarantees the truth which can never be fully reached merely by an spiritual appoach to God... There was a gap in the Tomist approach, but at that time the gap was not yet visible. By his genius, his power to take in almost everything, his power to mediate, his personal, even mystical, piety, Thomas was able to cover the gap, but the gap was there and had consequences reaching beyond what Thomas himself realized. This came out in Duns Scotus). P. TILLICH, A History of christian Thought from. Its Judaic and Hellenistic Origins to Existentialism, ed. By C. E. Braaten (New York 1968), pp. 186-187.

3. A. GARRIDO, «Autonomía o heteronomía...», p. 568. 
sin duda había de escandalizar y dar lugar después a resultados que todos conocemos en la historia de la filosofía moderna. Por otra parte los escolásticos comprendieron también que a Dios había que «introducirle» en este mundo (inmanencia), cuando nos hablaron de la presencia de Dios en él por esencia, presencia y potencia.

A Tillich le llama también la atención el problema de un encuentro auténtico con un Dios auténtico, con un Dios que, filosóficamente, no fuera una "piedra» o una "estrella», es decir, que no fuera un "otro» en el sentido de ser "al lado» de las demás cosas creadas, como el que parecía resultar de una demostración puramente racional; nos dice:

"Gilson puts it this way: 'It is indeed incontestable that in God essence and existence are identical. But this is true of the existence in which God subsists eternally in Himself; not of the existence to which our finite mind can rise when, by demostration, it estasblishes that God is'. It is obvious that this second concept of existence brings God's existence down to the level of that of a stone or a star, and it makes atheism not only possible, but almost unavoidable, as the later development has proved ${ }^{4}$.

Vale la pena analizar este texto por nosotros subrayado. Como se ve, Tillich, a propósito de esta distinción que hace Gilson entre la existencia de Dios en sí mismo, en su ser Dios sí mismo, y la existencia o ser de Dios como causa de las cosas que de él dependen, concluye que el Dios de la demostración puramente racional sería una "piedra» o una "estrella», es decir, un ser más "al lado», o "más allá», de los seres creados. Personalmente no creemos esta conclusión tan descabellada, ya que nos parece que se necesita el concepto de «creación», desconocido para la pura razón humana, y que precisamente es la raíz de la exigencia total y mutua entre la trascendencia y la inmanencia ${ }^{5}$.

No es, pues, de extrañar que a Tillich no le convenciera un acercamiento a Dios según una demostración racional aristotélica, sino más bien la postura agustiniana de una «inmediatez de la verdad en el hombre», como vamos a comprobarlo.

En su obra $A$ History of Christian Thought Tillich examina los enfoques distintos según las posturas agustiniana y aristotélico-tomista, haciendo observar que se puede trazar una línea de continuación que, partiendo de Agustín y mediante los franciscanos medievales y los filósofos de los siglos $X V I I I$ y XIX, llega hasta los filósofos de la religión de nuestros tiempos ${ }^{6}$. Per-

4. P. TILLICH, The two Types of Philosophy of Religion, en Theology of Culture, ed. by Robert C. Kinball (New York, Oxford University Press 1964), p. 18.

5. F. CASADO, «¿Es válida la demostración racional de la existencia de Dios a través de las "vías»?", en Estudio Agustiniano 5(1971) 627-640.

6. «We can trace a line of thought from Augustine to the philosophers of the seventeenth and eighteenth centuries, to the German classical philosophers, including Hegel, to the present 
sonalmente hemos podido comprobar un anillo de esta cadena en los filósofos agustinos más notables de los siglos XIII-XIV en lo que se refiere a lo espistemológico de la cuestión; de la misma manera lo hemos visto en otros filósofos como Luis Vives y Balmes ${ }^{7}$. En estas breves páginas queremos recordar que Tillich se encuadra también en una postura epistemológica de un innatismo de Dios en el hombre a base de una presencia inmanente y trascendente a la vez, algo básico para un encuentro racional con Dios mediante su inmediatez al hombre, optando así plenamente por la línea agustiniana ${ }^{8}$.

Tanto la tesis de J. Dourlay como los artículos del profesor A. Garrido ${ }^{9}$ nos recuerdan cómo Tillich se lamenta del conflicto existente entre las posturas agustiniano-franciscana por una parte y la aristotélico-tomista por otra ${ }^{10}$. Dourlay se mete de lleno con el pensamiento de Tillich intentando hacer ver las relaciones entre Dios y las criaturas (el hombre en particular) a través de unas categorías de esencia y existencia, implicando el concepto objetivo de esencia una "fuerte participación de lo inteligible y lo bueno de Dios», mientras que el concepto de existencia implicaría una "distancia», una especie de «culpa» o de «pecado». Todo esto en el aspecto teológico y relacionándolo con la posición de san Buenaventura. ¿En qué consistiría esta participación de Dios por parte del hombre? A pesar de lo extrañas que aparezcan las expresiones, desde que los filósofos del Renacimiento se plantearon el problema de la inmanencia de Dios en las criaturas, cosa inevitable si no queremos "colocar» a Dios en un lugar, se ha visto siempre la necesidad de ver a Dios más dentro de las criaturas que fuera. Porque ¿qué serían las criaturas fuera de Dios? Por eso Tillich prefiere decirnos que, en vez de decir que Dios es el primero de todos los seres, es posible decir que Dios es el poder ser en todas las cosas y sobre todas las cosas, el poder infinito de ser ${ }^{11}$. Es verdad que la

day philosophy of religion, to the extent it is not empirical philosophy of religion, which I think is a contradiction in terms, but a philosophy of religion which is based on the immediacy of the truth in every huma being". TILLICH, A History..., p. 104

7. F. CASADO, "La teoría de la memoria Dei en la tradición escolástica agustiniana», (extracto de la tesis doctoral, en Estudio Agustiniano 2(1967), pp. 85-109 y 347-363); «El apriorismo del conocimiento en Santo Tomás de Aquinon, en Est. Agust., 12(1977), pp. 493-509; "¿Apriorismo como contenido intelectual en Baimes, reflejo de la «memoria Dei» agustiniana?, en Avgustinus (1980), pp. 353-362; "Más acerca de la Memoria Dei», en Est. Agust., 8(1978)121126.

8. "I would say, almost unambiguosly, that I myself, and my whole theology, stand much more in the line of the Augustinian than in the Thomistic tradition». TILLICH, "A History...", p. 104.

9. J.P. DOURLAY, "Paul Tillich and Bonaventure. An evaluation of Tillich's claim to stand in the Agustinian-Franciscan Tradition». Leiden 1975; A. GARRIDO, "Presentación de Paul Tillich (1886-1965), en Est. Agust. 11(1976)381-421.

10. P. TILLICH, $\boldsymbol{A}$ History... pp. 180-186. En el apartado K del cap. III nos da Tillich la contraposición de los modos de pensar Agustiniano-franciscano y aristotélico-tomista partiendo ya de la distinta personalidad de los fundadores Francisco y Domingo, y retrotrayendo la cuestión a sus orígenes, Aristóteles y Agustín.

11. "Therefore, instead of saying that God is first of all being-itself, it is posible to say that 
noción de creación libraba a los escolásticos del panteísmo, pero no los autorizó a "colocar» a Dios al lado de la creación sino como «siendo» creación. Por eso el hombre, que es lo más noble de la creación por su ser consciente de su realidad procedente de Dios, es todo él una auténtica memoria Dei, un auténtico eco ontológico, imagen, reproducción de Dios. Habría, según Tillich, una coincidencia de opuestos: Dios-criaturas en una mutua exigencia de trascendencia-inmanencia, siempre que sea bien entendida. Porque, también dice Tillich, hay un peligro en esta frase que es pensar que el hombre es la situación central de esta trascendencia-inmanencia, como sucedió en la filosofía clásica romana y en la filosofía hegeliana ${ }^{12}$; ésta fue la preocupación del de Cusa, que derivó después en el panteísmo; pero al mismo tiempo habríamos de estar en guardia contra la convicción de que una pura filosofía podría librarnos de ese ateísmo panteísta.

Sobre esta presencia de Dios en el hombre, sobre este modo de ser memoria Dei el hombre por razón de la totalidad ontológica de su mismo ser como criatura, puede consultarse la obra de Tillich Systematic Theology; y para quien le resultase demasiado extensa, no diríamos pesada, su lectura recomendaríamos la obra ya citada de Dourlay, sobre todo el cap. III, y los artículos del profesor Garrido. En lo que sigue, nos vamos a limitar a la participación de Dios en el hombre a base de un innatismo «divino» en la esfera intelectual del mismo.

La «memoria Dei» según Tillich en el aspecto especifico de la teoría del conocimiento

Dejando a un lado esta inmanencia ontológica de Dios-creador en el hombre-criatura, vamos a fijarnos ahora en lo que, en particular afecta a la estructura de la razón humana, donde podemos ver un ejemplo más de esa presencia de Dios en el hombre desde el punto de vista del conocimiento humano y que encuadra a Tillich en la larga serie de los partidarios de un uinnatismo" intelectual.

Según Tillich, todo conocimiento está enraízado en el conocimiento de lo divino existente en nosotros. Citando a san Buenaventura, que afirmaba que el ser mismo es lo primero que aparece al entendimiento, nos dice Tillich que el ser es the basic statement about God, la manifestación fundamental acerca de Dios. Todo acto de conocimiento se hace en virtud de una «luz divina», de una "luz interior», en el alma humana, y mediante una presencia de Dios que él llama «teonomía». Pero citemos textualmente sus palabras:

«Bonaventura can say: 'Being itself is what first appears in the intellect,

he is the power of being in everything and above everything, the infinite power of being"s. Systematic Theologie, 1,236.

12. Ibid., p. 263. 
and being itself is the basic statement about God. This means that every act of cognition is made in the power of the divine light. The Franciscans said that this divine light, and these principles within us are uncreated, and we participate in them. Somehow this means that there is no such thing as secular knowledge. All knowledge is in some way rooted in the knowledge of the divine within us ${ }^{13}$.

El subrayado es nuestro, y lo hacemos para hacer hincapié precisamente en la dependencia metafísica del conocimiento humano respecto de ese contenido radical de conocimiento innato que ha sido denominado por los autores agustinos memoria Dei.

Pero el pensamiento de Tillich a este respecto se hace más incisivo al decirnos que, desde un punto de vista teológico, por el influjo de ese innato racional todo conocimiento humano, aun el científico, es implícitamente religioso. Continuamos copiando sus palabras:

"There is a point of identity in our soul, and this point precedes every special act of knowledge - about animals, plants, bodies, astronomy, mathematics - is implicitly religious. A mathematical proposition as well as a medical discovery is implicitly religious because it is posible only in the power of these ultimate principles which are the uncreated divine light in the human soul. This is the doctrine of the inner light, which was also used by the sectarian movements and by all the mystics during the Middle Ages and the Reformation period, and which in the last analysis underlies even the rationalism of the Enlightment. The rationalists were the philosophers of the innerlight, even though this light later on became out off from its divine ground. This attitude we call theonomous) ${ }^{14}$.

Para aclarar un poco este pensamiento de Tillich vamos a analizar algunas expresiones claves como éstas: «lo profundo de la razón» (the depth of reason), «autonomía», «heteronomía» y «teonomía» de la razón.

\section{La estructura de la razón}

Tillich comienza su Teología Sistemática hablándonos de la razón y de la revelación y hace una exposición de lo que entiende por la razón ontológica como estructura de la mente que la capacita para apoderarse de (grasping) y modelar o configurar (shaping) la realidad; esta razón es denominada razón subjetiva. Expone luego las cuatro maneras de interpretar esta razón subjetiva: a) como efecto dentro de un dogmatismo materialista; b) como creadora de un todo (Idealismo); c) como dualismo ontológicamente independiente con interdependencia funcional; $y d$ ) en forma de monismo subyacente, sin

13. A History..., p. 185.

14. Ibid. 
más explicación, a la razón subjetiva y objetiva. Por su parte Tillich, como teólogo, sin entrar ni salir en las discusiones sobre estas posturas, se detiene en consideraciones acerca de las dos funciones antes dichas, de «apoderarse de» y de "configurar» la realidad. Lo que aquí nos interesa a nuestro propósito es hacer hincapié en la función de "configuración» o modelación (shaping) de la realidad de la razón objetiva. Esta reacción de la razón subjetiva ya sería una especie de construcción de la realidad en cuanto conocida a base de la transformación verificada mediante esa "modelación", y que es una "forma» interna de ver las cosas; he aquí sus palabras:

"Shaping, in this context, has the connotation of transforming a given material into a GESTALT, a living structure which has the power of being ${ }^{15}$.

Relacionaríamos esto con ese gestaltismo de los modernos que hace que deba entenderse bien aquello de que «todo conocimiento empieza por los sentidos", que no puede ser entendido sin el ejercicio constructivo de los sentidos, es decir, aportando éstos algo innato a la objetividad del conocimiento. No diriamos que la forma triangular, por ejemplo, es vista por los ojos, cuyo objeto formal es el color o luz, pero no la forma como tal del triángulo...

Pero vamos a fijarnos particularmente en las expresiones claves del pensamiento de Tillich arriba citadas; la primera era "The depth of reason" que podríamos traducir por «lo profundo de la razón». Esta «profundidad» de la razón es algo que no es la razón y que la «precede» manifestándose a través de ella. Es algo así como la "sustancia», el "ser mismo» que se manifiesta en el logos del ser; es el "fondo» o "suelo» que es creador en toda creación racional, el "abismo de ser $y$ de significar», que fluye invadiendo la estructura racional de la mente y de la realidad ${ }^{16}$. Tenemos en todas estas expresiones como un contenido metafísico de la razón, no fuera de la razón aunque sí constituyendo un todo con ella; es un saber que la trasciende y no es un logos subjetivo de la pura razón, ni objetivo fuera de la razón; es un «mystical a priori» como nos dirá en otra parte ${ }^{17}$. Es un algo eterno que, sin identificarse con la mente, no puede verse completamente separado de ella. En el proceso del pensamiento este elemento eterno no está sujeto a la razón según lo entendiera Hegel, sino que vibra, pulsiona a través de todo el pensamiento humano de tal manera que no puede darse un pensamiento cualquiera que no presuponga esta verdad incondicional; también aqui preferimos reproducir sus palabras:

«But this eternal truth, this logos above fate, is not at man's disposal; it cannot be subjected, as Hegel thought it could, to the processes of human

15. Systematic Theologie, 1, p. 76

16. Ibid., l, p. 79.

17. Ibid., I, p. 9. 
thinking; it cannot be described or presented as the meaningfull world process. To be sure, this eternal logos does pulsate through all our thinking; there can be not act of thought without the secret presupposition of its inconditional truth" ${ }^{18}$.

Es claro que no se trata aquí de una concepción monístico panteísta sino de una luz interior, como nos ha dicho en algún texto anterior, de una especie de logos racional radical equivalente a algo que puede traducirse inmediatamente en unos principios últimos del juzgar y que él llama teonomía.

\section{Autonomía, heteronomía y teonomía}

También recordamos los artículos citados del profesor Garrido y la obra de Dourlay en lo que estas expresiones tienen de dependencia ontológica del ser total como criatura del Dios creador. Pero también aquí estas expresiones tienen un significado restringido y concreto al aspecto intelectual del conocimiento. En este sentido, autonomía o «razón autónoma» se define, según Tillich, como la razón que actualiza su estructura no atendiendo a la «depth of reason" ${ }^{19}$. Es decir, la autonomía es la ley de la razón subjetivo-objetiva pero que no tiene en cuenta las impresiones de la realidad dejadas a un lado (ungrasped impressions) así como el esfuerzo de modelación o configuración de esa realidad (unshaped striving). Esta autonomía entra en conflicto con la heteronomía en cuanto que ésta, a su vez, está mal entendida y se define como imposición desde fuera de la realidad de que "hay que apoderarse" y que hay que "configurar» ${ }^{20}$. Este conflicto derivado de estas concepciones equivocadas de autonomía y heteronomia, ha sido la historia del pensamiento en su desarrollo filosófico-teológico, y nos da la clave para su comprensión en toda su trayectoria desde los griegos hasta los tiempos modernos ${ }^{21}$. Según Tillich, no tiene lugar ese conflicto si se entienden bien la autonomía y la heteronomía. Cuando el "outside» de la heteronomía «is not merely outside», sino que representa al mismo tiempo «an element in reason itself, namely, the depth of reason", desaparece el origen del conflicto, siempre y cuando que la razón heterónoma no intente autoritariamente representar a la razón auténtica en contra de su actualización autonómica ${ }^{22}$. Es necesario que la autonomía y la heteronomía estén arraigadas en la teonomía para que ambas no yerren el camino ${ }^{23}$.

18. P. TILLICH, The Protestant Era, Chicago 1957, p. 14.

19. "Reason which affirms and actualizes its structure withourt regarding its depth is autonomous». Systematic Teol., I, p. 83.

20. "Heteronomy imposes a strange (heteros) law (nomos) on one or all of the functions of reason». Ib., p. 84.

21. Syst. Theol., I, p. 85-86. "The catastrophe of autonomous reason is complete. Neither autonomy nor heteronomy isolated and in conflict, can give the answern.

22. Ibid., p. 84.

23. Ibid., p. 85 . 
Pero ¿qué es esto que Tillich califica de teonomía? Ya del nombre se desprende como una presencia de lo divino en el hombre. $Y$ ¿qué es esto «divino»? Agustín había dicho que vemos «in rationibus aeternis», y es esto lo que cautiva la atención de Tillich que no duda en afirmar que se encuentra en la línea de Agustín. Tillich nos recuerda que el escepticismo fue lo que hizo que Agustín crease una nueva epistemología que da comienzo precisamente por la interioridad humana y no por el recurso a un mundo extraño ${ }^{24}$. Es en esta interioridad donde Agustín se refugia para encontrar la verdad ${ }^{25}$. Por otra parte, sigue diciendo Tillich, fue el escepticismo lo que hizo que Agustín se volviera a la Revelación. Con todo este análisis por delante Tillich cree ver una especie de filosofía religiosa, una teonomía de la mente, mediante la cual Dios, más bien que ser una conclusión de unas premisas, es antes que todas las conclusiones, y más bien las hace posibles ${ }^{26}$.

Tillich contempla los efectos catastróficos que ha causado en la filosofía griega el querer explicar el mundo a base de la pura razón filosófica ${ }^{27}$, y se admira de cómo Agustín ha sabido aprovecharse del neoplatonismo mediante el hombre interior como base para una nueva certeza de Dios ${ }^{28}$. Pero, mientras el neoplatonismo era una filosofía negativa, es decir, de escape hacia lo extramundano, en Agustín tenemos una filosofía positiva como contemplación de lo divino en la propia interioridad ${ }^{29}$. La epistemología que Agustín creó empieza por la interioridad humana en vez de partir de la experiencia del mundo exterior, no precisamente a base de la certeza del Yo, sino de la tenencia en sí de Dios ${ }^{30}$. Esta tenencia de Dios no ha de confundirse con la tenencia de la idea de Dios, como sucedería en Descartes; Dios es visto en el alma...; la consciencia no del Yo sino de la inmediatez de la tenencia de Dios...; Dios no

24. «Skepticismalso gave rise to a new doctrine of knowledge, a new epistemology, which Augustine created. It starts with the inner man instead of the experience of the external world». A History..., p. 108.

25. «Skepticism... had the effect of throwing Augustine upon himself to find therein the place of truth». A history..., p. 108.

26. «So from Augustine to Bonaventura we have a philosophy that is implicitly religious, or theonomous in which God is not conclusion from other premises, but prior to all conclusions, making them possible». A History..., p. 185.

27. "The heroic Greek attempt to build a world on the basis of philosophical reason came to a catastrophic end in skepticism». A History..., p. 108.

28. A History... p. 109.

29. «Neo-Platonism was a negative philosophy, a philosophy of escape from the world. The elevation of the soul out of material world into the ultimate is the meaning of Neo-Platonism. Augustine changed this enphasis; he dropped the idea of degrees, and instead used the NeoPlatonism for the immediate experience of the divine in everything, but specially in his soulı. $A$ History..., p. 109.

30. "The purpose and the way of knowledge are expressed in Augustine's famous words: "I wish to know God and the soul». "Nothing else?" "Nothinh at all». God and the soul! This means that the soul is the place where God appears to man. He wants to know the soul because only there can he know God, and in no other place». A History..., p. 11-112. 
es un ser en discusión..., más bien es nuestro a priori ${ }^{31}$. Tenemos aquí la resonancia de aquel «intimior intimo meò» de Agustín ${ }^{32}$. Aristóteles, pensador inductivo, va de abajo hacia arriba; Agustín, pensador intuitivo, de arriba hacia abajo ${ }^{33}$.

En conclusión, la teonomía significa, no la aceptación de la ley divina impuesta a la razón por una autoridad de arriba, es decir, desde afuera, sino la razón autónoma unida a su propia "profundidad» ${ }^{34}$. Situación teonómica significará autorrealización de la razón según sus leyes estructurales y en virtud del inexhaurible "suelo» o "profundo» (the depth) que en ella se encuentra ${ }^{35}$. Siendo por otra parte, Dios la ley tanto para la estructura de la razón como para su "suelo», ambas radican en él y tienen una unidad perfecta en la teonomía ${ }^{36}$. , de pasada, diremos que aquí indica Tillich que dada la imper-

31. "The difference is that in Descartes the self-centainty of the ego is the principle of mathematical evidence... whereas for Augustine the inner evidence is the immediacy of having God». A History..., p. 113.

32. Nos recuerda esta frase la situación de nuestro Unamuno en relación con Dios. Sabido es que, para este filósofo, un Dios, un alma inmortal, admitidos como el resultado de una demostración lógica no tienen razón de ser. En su obra El sentimiento trágico de la vida puede leerse lo siguiente: "La idea de Dios de la pretendida teodiceda racional, no es más que una hipótesis, como, por ejemplo, la idea de étern; de momento nos basta esta cita respecto de un encuentro con un Dios que no sea una mera idea, de tal manera que, para Unamuno, el problema de la existencia de Dios es un problema insoluble.- Desde luego, no otra cosa podría esperarse de un pensamiento existencialista. Pero no esto de lo que vamos a ocuparnos, sino de todo lo contrario, es decir, de la vivencia que Unamuno tiene de Dios, vivencia de la que parece un eco la postura de Tillich, sin que esto signifique que queramos decir que éste dependa de aquél. Oigamos algunas expresiones suyas: «El hombre ha ido a Dios por lo divino más bien que ha deducido lo divino de Dios» (p. 160). "Y este Dios, el Dios vivo, nuestro Dios, está en mí, está en ti, vive en nosotros, y nosotros vivimos, nos movemos y somos en él. Y está en nosotros por el hambre que de él tenemos, por el anhelo, haciéndose apetecer. $Y$ es el Dios de los humildes, porque Dios escogió lo necio del mundo...» (p. 180). «El Dios de que tenemos hambre es el Dios a que oramos, el Dios del Paternoster, de la oración dominical; el Dios a quien pedimos, ante todo y sobre todo, démonos o no de esto cuenta, que nos infunde fe, fe en él mismo, que haga que creamos en él, que se haga él en nosotros..." (lb.). "Y tal es el Dios del amor... Ese en que crees, lector, ese es tu Dios, el que ha vivido contigo en ti, y naçió contigo y fue niño cuando eras tú niño, y fue haciéndose hombre, según tú te hacias hombre...) (Ibid.). "No es, pues, necesidad racional, sino angustia vital, lo que nos lleva a creer en Dios. $Y$ creer en Dios es, ante todo y sobre todo, he de repetirlo, sentir hambre de Dios, hambre de divinidad, sentir su ausencia y vacío, querer que Dios exista) (p. 187). "Creer en Dios es anhelar que lo haya, y es además conducirse como si lo hubiera; es vivir de ese anhelo y hacer de él nuestro íntimo resorte de acción» (p. 187-188). "A ese Dios cordial y vivo se llega, y se vuelve a él cuando por el Dios lógico o muerto se le ha dejado, por camino de fe y no por convicción racional o matemática» (p. 189).

Creo basten estos textos para ver la gran analogía existente, a este respecto con Tillich cuando se trata no de una tenencia de la idea de Dios en nosotros, sino de la tenencia de Dios.

33. A History..., p. 111.

34. "Theonomy does non mean the acceptance of a divine law inposed on reason by a highest authority; it means autonomous reason united with its own depth». Syst. Theol., I, p. 85.

35. Ibid.

36. Ibid. 
fección de este orden existencial en que se mueven las criaturas, el resquebrajamiento que existirá entre esos dos elementos debe ser subsanado por la revelación (No se olvide que Tillich está escribiendo su Teología Sistemática).

Lo que en todo esto nos interesa es que ese "ground», ese "depth» en y de la razón es lo «innato» constitutivo de esa memoria Dei admitida también por Tillich.

\section{Fundación teonómica de la ley moral}

Un caso concreto de la teonomía como presencia de Dios natural en el alma, y que se ha considerado como característico de la memoria Dei en el hombre, ha sido la ley moral; y a Tillich no se le pasó por alto. En su tercer volumen de la Teología Sistemática dedica unas páginas a considerar "to stablish a theonomous foundation for the moral law». La cuestión es planteada en términos similares a la cuestión general de autonomía, heteronomía y teonomía a que antes hemos hecho alusión. También la ley moral, los diez mandamientos, universalmente válidos, entran en conflicto al tratarse de situaciones circunstanciales en las existencias humanas. Pues bien, la superación del conflicto entre lo esencial y universal de la ley moral se verifica mediante la ley del amor, efecto de la Presencia Espiritual, que no es voluntad del hombre, sino también gracia, don que potencia la dirección de la realización moral de la persona corrigiendo lo conflictivo entre lo esencial y lo existencial en el hombre, fundamentando así teonómicamente en él el orden moral. No olvidemos que se trata aquí del teólogo que está hablando de una ética que, siendo natural como estructura del hombre moral, sin embargo en situaciones concretas no será auténtica sin la ayuda de eso que llama Presencia Espiritual que corregirá las desviaciones y fundamentará la validez incondicional de la moral imperativa:

«The many forms of ethics without Spiritual Presence are judged by the fact that they cannot show the power of motivation, the principle of choice in the concrete situation, the inconditional validity of the moral imperative. Love can do it, but love is not a matter of man's will. It is a creation of the Spiritual Presence. It is grace» ${ }^{37}$.

Concluiríamos estas breves páginas diciendo que esa memoria Dei como presencia de Dios en el hombre y que fue llamada también por uno de los primeros entusiastas de la misma "tirante ontológico» que unía dos realidades distintas, en Tillich se acerca más también a una concepción de coincidencia de lo Infinito y lo finito como inmanencia ontológica del Creador en la criatura en la que se verifica una teonomía vivencial que Tillich considera también en el orden sobrenatural.

Fidel CASADO

37. Syst. Theol., III, 274-275. 